\title{
Multiuser Automatic Modulation Classification for Cognitive Radios using Distributed Sensing in Multipath Fading Channels
}

\author{
Munawwar M. Sohul, Barathram Ramkumar*, Tamal Bose \\ Wireless @ Virginia Tech \\ Bradley Dept. of Electrical and Computer Engineering \\ Virginia Polytechnic Institute and State University \\ Blacksburg, Virginia 24060 \\ *School of Electrical and Computer Engineering, IIT-Mandi, India \\ E-mail:mmsohul@vt.edu, *barathram@iitmandi.ac.in, tbose@vt.edu
}

\begin{abstract}
Automatic Modulation Classifier (AMC) is an important component of a Cognitive Radio (CR) architecture that helps in better utilization of the spectrum. The successful implementation and employment of various cognitive radio services are largely dependent on the modulation classification performance of the cognitive radio terminals. AMC in literature is mostly developed for classifying the signal transmitted by a single user. Multiuser AMC, as the name suggests, simultaneously classifies signals transmitted by multiple users, while existing approaches rely on centralized processing. In this paper, we propose a fourth order cumulant based multiuser distributed AMC that can perform well even in a multipath fading environment. The proposed algorithm uses spatially distributed CRs cooperating with each other, which results in improved classification performance. Simulation results are provided to illustrate the promising results yielded by the proposed algorithm.
\end{abstract}

Keywords: Cognitive radio, multiuser AMC, distributed classification, higher order cumulants, multipath fading

\section{INTRODUCTION}

Cognitive radio, originally introduced by Mitola [1], has emerged as a key enabling technology which provides licensed users with the ability to share the wireless channel in an opportunistic way [2]. AMC is the automatic recognition of modulated signals present in a particular frequency band. AMC is an important component of cognitive radio that improves spectral efficiency by adapting transmission and reception according to the spectral environment. CRs are basically intended to form an ad-hoc network known as a Cognitive Radio Network (CRN) [3], which has potential military and commercial applications. In military and public safety applications, the CRs must be capable of performing fixed and on-the-move communications between highly diverse elements in a harsh environment which may also be susceptible to jamming attacks and malicious interference [4]. For the secure and reliable operation of a CRN, CRs must be able to identify all users in the frequency band simultaneously.
AMC can be broadly classified into two categories: Likelihood based and Feature based [5]. Feature based AMCs are widely used because of easy implementation and better performance. Some of the widely used features are higher order statistics, cyclostationary features, wavelet features, and signal constellation. A lot of work has been reported in the literature on classifying signals from a single user, but multiuser AMC has not yet been investigated to a reasonable extent. The idea of multiuser AMC using a fourth order cumulant based approach has been recently proposed in [6]. However, it assumes that the number of transmitting users is known and all the users transmit at the same power over an AWGN channel, which is not true in a normal cognitive radio setup. Also, the method in [6] does not identify the exact modulation schemes used by the transmitting users but rather identifies the possible family of modulation schemes that might be present in the frequency band.

In this paper, we propose a novel multiuser distributed AMC based on normalized fourth order cumulants that can identify the exact modulation schemes used by multiple transmitting users in a frequency band. The proposed AMC is developed for actual multipath fading environments and no assumption on the transmission power of the users is made. In the proposed multiuser AMC, we use multiple antennas for reception, whereas only a single receiving antenna is used in [6]. By using multiple antennas at the receiver, the CR can identify the number of transmitting users, which is generally not possible while using a single antenna receiver. Also, by using multiple antennas, the CR can harness the flexibility offered by traditional multi-input multi-output (MIMO) communication schemes separately from classifying the signals from multiple users.

The normalized cumulant value based single user AMC was first proposed in [7]. The multipath channel drives the cumulant value of the transmitted signal to zero [7] and hence 
severely affects the performance of the cumulants based AMC. In [8], [9] a robust cumulant based single user AMC was developed for multipath fading channels. The approach in [8] involves estimating the multipath channel and using the estimated channel information to improve the performance of the AMC. However, channel knowledge or a pilot sequence for estimating the channel is not available in a CR scenario. Therefore, one needs to estimate the channel blindly. Most of the blind multiuser channel identification algorithms reported in the literature are batch processing algorithms. The high computational overhead involved in computing the inverses of a large correlation matrix as a part of these algorithms is not suited for CRs in rapidly varying channel conditions. In this paper, we will use a recursive channel estimation scheme that can track rapidly changing channel conditions for the single receiver AMC scheme proposed in our previous work $[10,11]$. The proposed multiuser AMC consists of two major blocks: a signal processing block and a classifier block. In the signal processing block, the normalized fourth order cumulant of the received signal and the multiuser channel impulse response are estimated and used to determine the normalized cumulant value of each transmitting user. These estimated cumulant values are finally fed to the classification unit to identify the modulation schemes employed by users.

In this paper, signal detection and modulation classification is performed in a distributed manner to take advantage of radio signal variability [12]. There has been a relatively rich history of research in the area of distributed detection and estimation theory [13, 14]. A number of papers have proposed, and addressed, distributed signal detection in the context of cognitive radios [15-17]. Recently, some works have focused on distributed signal classification in sensor networks. An optimized solution is proposed in [18] that incorporates distributed detection to the framework of LRT-based modulation classification. In [12], a distributed cyclic spectrum feature based modulation classification algorithm is proposed where a centralized fusion center makes a final decision based on locally estimated classifications using the Gauss-Seidel iteration. In [19], distributed feature based modulation classification in the context of wireless sensor networks is presented where classification performance is improved by exchanging suitable information among the single hop neighbors.

All of the above works either consider a single transmitting user or a single receiving node to analyze the classification performance. In this paper, we try to analyze the classification performance assuming multiple transmitting users and a spatially distributed CRN where all the CRs operate in a cooperative manner. The distributed setup comes into play as the cumulants are estimated from the received samples. CRs exchange their local version of the sample average estimation with their neighbors. Individual sensors update their cumulant estimation using information from their neighbors in an iterative fashion.
The paper is organized as follows. In Section II we present the theory behind multiuser AMC. The channel model and the assumptions made are also presented in this section. Section III presents a discussion of distributed feature based AMC. In Section IV, we present the multiuser distributed modulation classification algorithm. Simulation results are presented in Section $\mathrm{V}$ followed by the conclusion.

Notation: (.) ${ }^{\mathrm{T}}$ denotes the usual transpose operation; (.) ${ }^{*}$ or $(.)^{\mathrm{H}}$ denotes the complex conjugate transpose; $I_{m}$ denotes the identity matrix of dimension $m \times m ; O_{m \times n}$ denotes the zero matrix of dimension $m \times n$; $|()$.$| denotes the absolute value of$ the variable; $E($.$) denotes the statistical expectation. Whenever$ it is clear from the context, the dimensions of $I$ or $O$ will be omitted for simplicity of presentation and can be inferred from the context.

\section{ChANNEL MODEL AND PRELIMINARIES}

In this section, the underlying theory behind the proposed cumulant based multiuser AMC is provided. We begin our discussion by presenting the channel model and the assumptions in the channel model

\section{A. Channel model and assumptions}

In order to classify the signal from multiple users simultaneously, a receiver should have multiple antennas. Let $l$ be the number of transmitting users and $m$ be the number of receiving antennas; it is required that $m>l$. The above condition is required for the blind estimation of the multiuser channel. Usually in a CR scenario, $l$ is not known, but there are methods available in the literature for estimating $l$ using multiple receiving antennas (see example in [20]). The multipath channel between the $j^{\text {th }}$ user and the $i^{\text {th }}$ receiving antenna is denoted as $h_{i j}\left(z^{-1}\right)$ and is given by

$$
h_{i j}\left(z^{-1}\right)=h_{i j}(0)+h_{i j}(1) z^{-1}+\ldots+h_{i j}(L) z^{-L},
$$

where $L$ is the number of multipath components, $z^{-1}$ is the unit delay operator, and $h_{i j}(k)$ (for $k=1, \ldots, L$ ) is the fading coefficients of the corresponding multipaths. The overall system can now be represented by the following model

$$
\begin{aligned}
& y(i)=x(i)+w(i), \quad i=0,1,2, \ldots \\
& x(i)=H\left(z^{-1}\right) s(i)
\end{aligned}
$$

where $s(i)$ is the $l \times 1$ transmission vector whose elements $s_{k}(i)$ ( $k=1,2 \ldots l$ ) denote the $k^{\text {th }}$ transmitting user, $y(i)$ is the $m \times 1$ reception vector whose elements $y_{k}(i)(k=1,2 \ldots m)$ denote the received signal at the $k^{\text {th }}$ receiving antenna, $w(i)$ denotes the $m$ $\times 1$ noise vector, and $H\left(z^{-1}\right)$ is given by

$$
H\left(z^{-1}\right)=\left[\begin{array}{ccc}
h_{11}\left(z^{-1}\right) & \cdots & h_{1 l}\left(z^{-1}\right) \\
\vdots & \ddots & \vdots \\
h_{m 1}\left(z^{-1}\right) & \cdots & h_{m l}\left(z^{-1}\right)
\end{array}\right]
$$


We make the following assumptions regarding the system model (2).

Assumption A1: $\operatorname{rank}\left[H\left(z^{-1}\right)\right]=l$, for all complex $z \neq 0$, i.e. $H\left(z^{-1}\right)$ is irreducible.

Assumption A2: $s(k)$ is zero mean, spatially independent, and temporally white.

Nonidentity correlation matrices are absorbed into $H\left(z^{-1}\right)$, i.e., the transmission power of the users can be different. $\sigma_{w}^{2}$.

Assumption A3: $w(k)$ is zero mean Gaussian with variance

Assumption A1 is verified with probability one for any practical MIMO wireless channel with reasonable spatial diversity and hence for our CR scenario this assumption is valid. Assumption A2 implies that signals transmitted by two different users are uncorrelated. Assumption A3 implies that that the noise vector is uncorrelated and variance $\sigma_{w}^{2}$ is known. In general, $\sigma_{w}^{2}$ is not known, but there are several methods in the literature for estimating it.

\section{B. Cumulant based multiuser AMC}

In this section, we provide the basic theory behind the proposed cumulants based multiuser AMC. Cumulant features were first considered for the AMC in [7].

In a multiuser scenario, the received signal at an antenna array is a superposition of signals from many users. Cumulant features are considered in this case because of the following additive property

$$
C_{40_{u+v}}=C_{40_{u}}+C_{40_{v}}
$$

where $u$ and $v$ are independent sequences. The normalized fourth order cumulant of a received signal is defined as

$$
\begin{aligned}
\tilde{C}_{40_{v}} & =\frac{C_{40_{v}}}{\left(C_{21 v}\right)^{2}} \\
\text { where } \quad C_{21_{v}} & =E\left(|v|^{2}\right)
\end{aligned}
$$

The value of $C_{40_{v}}$ can be estimated from the sample estimates of the corresponding moments [7] and we denote it as $\tilde{C}_{40_{v}}$. The $C_{40_{v}}$ value for most modulation schemes is unique and hence can be used as a feature for classification. The theoretical values of cumulants for different modulation schemes are tabulated in [7]. Since multiple receiving antennas are used, the received signal at the $i^{\text {th }}$ receiving antenna due to multiple transmitting users is given by

$$
y_{i}(n)=h_{i 1}\left(z^{-1}\right) s_{1}(n)+\cdots+h_{i l}\left(z^{-1}\right) s_{l}(n)+w_{i}(n)
$$

Using the relationship in (5), the value of the fourth order cumulant for $y_{i}$ is given by

$$
C_{40_{y_{i}}}=C_{40_{s_{1}}} \gamma_{i 1}+\cdots+C_{40_{s}} \gamma_{i l}
$$

where $\gamma_{i j}=\sum_{k=0}^{L-1}\left|h_{i j}(k)\right|^{4}$
$C_{21_{y_{i}}}=C_{21_{s_{1}}} \rho_{i 1}+\cdots+C_{21_{s_{l}}} \rho_{i l}+\sigma_{w}^{2}$

where $\quad \rho_{i j}=\sum_{k=0}^{L-1}\left|h_{i j}(k)\right|^{2}$

Assumption A2 implies $C_{21_{s_{i}}}=1($ for $i=1, \ldots, l)$, i.e., transmitted signals are of unit energy. It should be noted that non unit energy signals are converted to unit energy by absorbing the scaling factor into the channel matrix $H\left(z^{-1}\right)$. Thus (11) can be written as

$$
C_{21_{y_{i}}}=\rho_{i 1}+\cdots+\rho_{i l}+\sigma_{w}^{2}=\Delta_{i}+\sigma_{w}^{2}
$$

Then the normalized $4^{\text {th }}$ order cumulant for $y_{i}$ is given by

$$
\tilde{C}_{40 y_{i}}=\frac{C_{40 y_{i}}}{C_{21 y_{i}}{ }^{2} \sigma_{w}^{2}}=\sum_{j=1}^{l} \frac{\gamma_{i j}}{\Delta_{i}{ }^{2}} \tilde{C}_{40_{s_{i}}}
$$

Extending the above equation to all receiving antennas

$$
\left[\begin{array}{c}
\tilde{C}_{40_{y_{1}}} \\
\vdots \\
\tilde{C}_{40_{y_{m}}}
\end{array}\right]=\left[\begin{array}{ccc}
\frac{\gamma_{11}}{\Delta_{1}{ }^{2}} & \cdots & \frac{\gamma_{1 l}}{\Delta_{1}{ }^{2}} \\
\vdots & \ddots & \vdots \\
\frac{\gamma_{m 1}}{\Delta_{m}{ }^{2}} & \cdots & \frac{\gamma_{m l}}{\Delta_{m}{ }^{2}}
\end{array}\right]\left[\begin{array}{c}
\tilde{C}_{40_{x_{1}}} \\
\vdots \\
\tilde{C}_{40_{x_{l}}}
\end{array}\right]
$$

or $\quad \vec{C}_{40_{y}}=B \vec{C}_{40_{x}}$

The cumulant values of the signal transmitted by different users can be obtained by solving (15). The extracted cumulant features are then used for classification. Assumption A1 implies that the entries of $H\left(z^{-1}\right)$ do not have any common factors, i.e., each entry of $H\left(z^{-1}\right)$ is a unique polynomial. Under this assumption, the matrix $B$ always has full column rank with probability 1 . Hence the solution to (15) is given by

$$
\vec{C}_{40_{x}}=\left(B^{H} B\right)^{-1} B^{H} \vec{C}_{40 y}
$$

In order to compute the $B$ matrix, we require knowledge of the channel matrix $H\left(z^{-1}\right)$. In a CR scenario, $H\left(z^{-1}\right)$ is not known and needs to be estimated blindly. In our earlier work [11], we proposed a recursive multiuser channel estimation algorithm in the cognitive radio context. We will use that blind recursive estimation to acquire the required knowledge of the channel matrix $H\left(z^{-1}\right)$. The estimated $H\left(z^{-1}\right)$ will be used calculate the $4^{\text {th }}$ order cumulants.

\section{Distributed Feature Based AMC}

The local cumulants are the expected values of different powers of the received sequence. In practice, these expectations are replaced by sample averages over the received symbols. Sample estimates of the $4^{\text {th }}$ and $2^{\text {nd }}$ order cumulants are given by

$$
C_{40_{s_{j}}}=\frac{1}{N} \sum_{n=1}^{N} r_{j}^{4}(n)-3\left(C_{20_{s_{j}}}\right)^{2}
$$

where $\quad C_{20_{s_{j}}}=\frac{1}{N} \sum_{n=1}^{N} r_{j}^{2}(n)$

and $\quad C_{21_{s_{j}}}=\frac{1}{N} \sum_{n=1}^{N}\left|r_{j}(n)\right|^{2}$

Similarly, the second order cumulant for $y_{i}$ is given by 
Here $r_{j}(n)$ is the received signal and $N$ is the total number of samples under consideration. While calculating the sample estimates of the second order cumulants, we need to consider the effect of the noise process. A local estimate of $\frac{N_{0}}{2}$ is obtained and subtracted from the second order cumulant estimates.

The classification performance depends on how accurately the cumulants can be estimated. This involves reducing the variance of cumulant estimates by acquiring longer sequences of symbols. The strong law of large numbers guarantees that $C_{21_{s_{j}}} \rightarrow C_{21}$ and $C_{40_{s_{j}}} \rightarrow C_{40}$ as $N \rightarrow \infty$ with probability one. However, CRs can typically observe only a short part of the transmitted sequence. To reduce the resultant high-variance of local cumulant estimates it is thus critical for sensors to share information when computing averages in (19) and (20).

$$
\begin{aligned}
& \text { Let, } \quad p_{40_{s_{j}}}=\frac{1}{N} \sum_{n=1}^{N} r_{j}^{4}(n), \\
& \qquad p_{20_{s_{j}}}=\frac{1}{N} \sum_{n=1}^{N} r_{j}^{2}(n), \\
& \text { and } \quad p_{21_{s_{j}}}=\frac{1}{N} \sum_{n=1}^{N}\left|r_{j}(n)\right|^{2} .
\end{aligned}
$$

The central limit theorem implies that for large values of samples the distribution of the sample averages approximates a Gaussian distribution. Also, an estimate of the sample average that combines all received symbols across CRs has a variance smaller than that of a local estimate. The local averages can be expressed as

$$
p_{21_{s_{j}}}=\hat{p}_{21}+g_{21_{s_{j}}}, j=1,2, \ldots, J
$$

where $g_{21_{s_{j}}}$ is a complex white zero mean Gaussian random variable (similarly we can estimate $p_{40_{s_{j}}}$ ). The maximum likelihood estimator of $\hat{p}_{21}$ can be found in closed form as given by

$$
\hat{p}_{21_{M L}}=\frac{1}{J} \sum_{j=1}^{J} p_{21_{s_{j}}}
$$

Finding $\hat{p}_{21_{M L}}$ involves computing an average of the local estimates $p_{21_{s_{j}}}$. For the computation of the averages distributively using a Cognitive Radio Network (CRN), we consider the approach taken by [19]. Here, an approach based on the method of multipliers (MoM) is used because of its added resilience to noise and its fast convergence [21]. The average of the local estimates $\hat{p}_{21_{M L}}$ is, in fact, the solution of the following relation

$$
\min _{\hat{p}_{21_{M L}}} \frac{1}{2} \sum_{j=1}^{J}\left\|\hat{p}_{21}-p_{21_{s_{j}}}\right\|^{2}
$$

Problem (23) cannot be solved in a distributed fashion because of the communication constraints imposed by the topology of the CRN. For this reason, local variables $\hat{p}_{21_{s_{j}}}$ are introduced together with equality constraint to rewrite (23) as

$$
\begin{aligned}
& \min _{21_{j}} \frac{1}{2} \sum_{j=1}^{J}\left\|\hat{p}_{21_{j}}-p_{21_{s_{j}}}\right\|^{2} \\
& \text { s.t. } \hat{p}_{21_{j}}=\hat{p}_{21_{i}} \text { for } j=1,2, \ldots, J, \quad \forall_{i} \in N
\end{aligned}
$$

The equality constraints force all CRs to consent on the solution of (21). The MoM uses the augmented Lagrangian to construct an iterative algorithm solving (24). A Lagrange multiplier vector $\gamma_{j}^{i} \in \boldsymbol{R}^{2}$ is introduced for each of the equality constraints in (24). When $\mathrm{U}:=\left\{\gamma_{j}^{i}, j=1,2, \ldots, J, i \in N_{j}\right\}$ denotes the set of all multipliers, the augmented Lagrangian is given by

$$
\begin{gathered}
\left.L\left(\hat{p}_{21_{j}}\right\}_{j=1}^{J}, \mathrm{U}\right)=\frac{1}{2} \sum_{j=1}^{J}\left\|\hat{p}_{21_{j}}-p_{21_{s_{j}}}\right\|^{2}+ \\
\sum_{j=1}^{J} \sum_{i \in N_{j}} \gamma_{j}^{i T}\left(\hat{p}_{21_{j}}-\hat{p}_{21_{i}}\right)+\sum_{j=1}^{J} \sum_{i \in N_{j}} \frac{\xi}{2}\left\|\hat{p}_{21_{j}}-\hat{p}_{21_{i}}\right\|^{2}
\end{gathered}
$$

where $\xi>0$ is a weight introduced to penalize the quadratic term and to trade off convergence rate for accuracy. The Mom based algorithm solves (24) by minimizing (25) one variable at a time. Per iteration l, each CR updates its local summary statistics

$$
\hat{p}_{21_{j}}{ }^{(l+1)}=\frac{p_{21_{j}}+\sum_{i \in N_{j}}\left(\gamma_{i}^{j(l)}-\gamma_{j}^{i(l)}+2 \xi \hat{p}_{21_{j}}{ }^{(l)}\right)}{1+2 \xi\left|N_{j}\right|}
$$

and updates its Lagrange multipliers

$$
\gamma_{j}^{i(l+1)}=\gamma_{j}^{i(l)}+\xi\left(\hat{p}_{21_{j}}{ }^{(l+1)}-\hat{p}_{21_{i}}{ }^{(l+1)}\right)
$$

The MoM based algorithm for computing the average of the local estimates is given bellow:

1. All CRs randomly initialize their Lagrange multipliers $\mathrm{U}^{(0)}$ and local estimates $\hat{p}_{21_{j}}{ }^{(0)}, j=1,2, \ldots, \mathrm{J}$;

2. Per iteration $l$, each CR broadcasts its multipliers $\gamma_{j}^{i(l)}$ and local estimates $\hat{p}_{21_{j}}{ }^{(l)}$ to its neighboring nodes $i \in N_{j}$;

3. Then all CRs update their estimates $\hat{p}_{21_{j}}{ }^{(l)}$ via (26);

4. Each CR broadcasts its new estimates $\hat{p}_{21_{j}}{ }^{(l+1)}$ to its neighbors $i \in N_{j}$;

5. Finally, each CR updates its multipliers $\gamma_{j}^{i(l)}$ via (27).

The vector of normalized cumulants is used in (9) to calculate the normalized cumulant of that particular CR. These 
cumulant values are used to classify the unknown modulation format. Note that each CR is now able to make a decision about the modulation format on its own.

\section{ClassificATION AlgORITHM}

In this section, we present the step-by-step procedure for performing multiuser distributed AMC. The multiuser distributed AMC is obtained by applying the estimated channel from our previous work [11] and the estimated sample average through cooperation in a distributed setup on the classification algorithm developed in Section II.

Step 1. Estimate the channel $H\left(z^{-1}\right)$ using the blind recursive channel estimation algorithm proposed in [11].

Step 2. Estimate the B matrix of (18) using the estimated channel $H\left(z^{-1}\right)$.

Step 3. Calculate the estimate of the $4^{\text {th }}$ and $2^{\text {nd }}$ order cumulants using (18), (20), and the MoM based algorithm presented in Section III.

Step 4. The vector of normalized cumulants estimated in Step 3 is used in (9) to calculate the normalized cumulant of that particular CR due to the transmission of multiple users.

Step 5. Use matrix B from Step 3 and the normalized cumulants from Step 4 in (17) to estimate the cumulant features of all the transmitted sequences.

Step 6. This is the final step where we classify the signals from multiple users using the estimated cumulant feature vector $\vec{C}_{40_{x}}$.

Step 7: Continuously monitor the channel coefficients using the blind recursive algorithm. If the channel conditions change drastically, then repeat Step 3 to estimate the new channel impulse response.

\section{Simulation RESUltS}

In this section, we present the performance of the proposed algorithm to classify signal modulation from multiple users in a distributed setup. Our primary interest was to observe the improvement achievable by introducing cooperation among CR users in a CRN over a single CR user scenario. We also tried to observe the impact of an observation period on the performance of the proposed multiuser AMC system.

The performance measure used is the probability of correct classification $P_{C}$, i.e. the probability that the multiuser AMC successfully classifies the set of modulations coming from multiple transmitting users. Suppose that there are $l$ transmitting users, $M$ modulation schemes which are denoted as $\alpha=\left\{\alpha_{1}, \alpha_{2}, \ldots, \alpha_{M}\right\}$, and k CR receivers cooperating with each other. Then there are $L_{1}=M^{l}$ possible transmission scenarios denoted as $D=\left\{d_{1}, d_{2}, \ldots, d_{L_{1}}\right\}$. The probability of correct classification $P_{C}$ is defined as

$$
P_{C}=\sum_{i=1}^{L_{1}} P\left(d_{i} \mid d_{i}\right) P\left(d_{i}\right)
$$

where $P\left(d_{i}\right)$ is the probability that the particular transmission scenario occurs and $P\left(d_{i} \mid d_{i}\right)$ is the correct classification probability when scenario $d_{i}$ has been transmitted. For the simulation, we assume $P\left(d_{i}\right)=\frac{1}{L_{1}}, \forall_{i}$, where all scenarios are equally probable. In all the simulations, the signal-to-noise ratio (SNR) is defined as

$$
S N R=\frac{\sum_{i=1}^{m} E\left(\left|x_{i}\right|^{2}\right)}{\sum_{i=1}^{m} E\left(\left|w_{i}\right|^{2}\right)}
$$

In this part of the simulation we considered multipath fading channels. The blind recursive algorithm from [11] was used to estimate the channel. The multiuser distributed AMC algorithm was simulated to observe the improvement in classification performance over a single CR user scenario. The probability of correct classification vs. SNR in $\mathrm{dB}$ is presented in Fig. 1 for both cases. As can be seen from Fig. 1, introducing cooperation among CRs in a spatially distributed CRN yields improved performance when making use of the available diversity of the distributed setup.

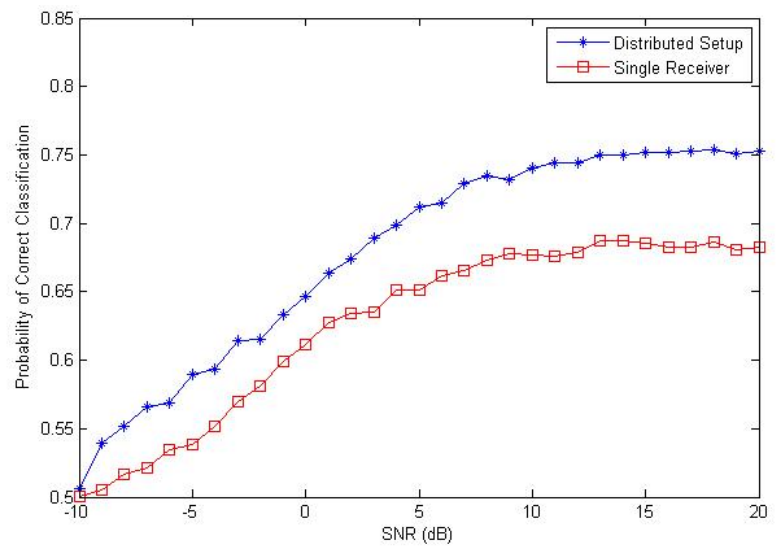

Fig. 1. Performance of the multiuser AMC in a distributed setup: Multipath fading channel, $\{$ BPSK, QPSK, 8-PSK\}, $\mathrm{T}=1000$.

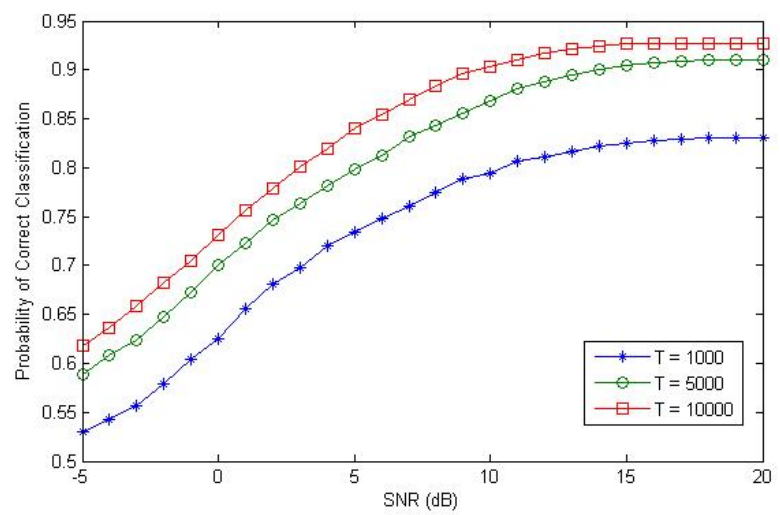

Fig. 2. Performance of the multiuser AMC in a distributed setup: Multipath fading channel, \{BPSK, QPSK, 8-PSK\}, $\mathrm{T}=1000,5000,10000$. 
To observe the impact of the observation period on classification performance in the case of multipath fading channels, a simulation was run for different numbers of samples. The resulting probability of correct classification vs. SNR graphs is given in Fig. 2. From Fig. 2, it can be seen that the AMC performs well when the number of observations is increased.

\section{CONCLUSION}

In this paper, we presented a novel cumulant based multiuser distributed AMC for fading channels. The proposed AMC does not require any prior knowledge about the channel and hence is suitable for CR applications. We presented the performance of the proposed algorithm to classify signal modulation from multiple users in a distributed setup. Our primary interest was to observe the improvement achievable by introducing cooperation among CR users in a CRN over a single CR user scenario. We also tried to observe the impact of an observation period on the performance of the proposed multiuser AMC system.

\section{REFERENCES}

[1] J. Mitola III, "Cognitive radio: An integrated agent architecture for software defined radio," Dissertation, Royal Inst. Technol. (KTH), Stockholm, Sweden, 2000.

[2] S. Haykin, "Cognitive radio: Brain-empowered wireless communications," IEEE J. Select. Areas Commun, vol. 23, pp. 201-220, 2005.

[3] R. W. Thomas, D. H. Friend, L. A. DaSilva, and A. B. MacKenzie, “ Cognitive networks: adaptation and learning to achieve end-to-end performance objectives,” IEEE Commun Mag, vol. 44, pp. 51-57, 2006.

[4] B. Perlman, J. Laskar, and K. Lim, "Fine-tuning commercial and military radio design," IEEE Microwave Mag, vol. 9, pp. 95-106, 2008.

[5] O. A. Dobre, A. Abdi, Y. Bar-Ness, and W. Su, "Survey of automatic modulation classification techniques: Classical approaches and new trends," IET Communication, vol. 1, no. 2, pp. 137-156, Apr. 2007.

[6] M. Zaerin, B. Seyfe, and H. R. Nikoofar, "Multiuser modulation classification based on cumulants in AWGN channel," Submitted to IEEE Transaction on Communications, http://arxiv.org/abs/0908.2117.
[7] A. Swami and B. M. Sadler, "Hierarchical digital modulation classification using cumulants,” IEEE Trans. Commun., vol. 48, no. 3, pp. 416-429, Mar. 2000.

[8] S. Xi and H.-C. Wu, "Robust automatic modulation classification using cumulant features in the presence of fading channels," in Proc. IEEE Wireless Communications and Networking Conference, Apr. 2006, vol. 4, pp. 2094-2099.

[9] H.-C. Wu, Y. Wu, J. C. Principe and X. Wang, "Robust switching blind equalizer for wireless cognitive receivers," IEEE Trans. Wireless Commun, vol. 7, no. 5, pp. 1461-1465, May 2008.

[10] M. S. Radenkovic, T. Bose, and R. Barathram, "Recursive blind equalization of MIMO systems," in Proc. SDR Forum, Nov. 2008.

[11] B. Ramkumar, T. Bose, and M. S. Radenkovic, "Robust Multiuser Automatic Modulation Classifier for Multipath Fading Channels", New Frontiers in Dynamic Spectrum, 2010 IEEE Symposium on, pp. 1-10, 69 April. 2010.

[12] W. C. Headley, J. D. Reed, and C. R. C. M. da Silva, "Distributed Cyclic Spectrum Feature-based Modulation Classification", IEEE Wireless Communications and Networking Conference, , pp. 1200-1204, 15 April. 2008.

[13] P. K. Varshney, Distributed Detection and Data Fusion. Englewood Clifffs, NY: Springer, 1997.

[14] J. N. Tsitsiklis and M. Athans, "On the complexity of decentralized decision making and detection problems," IEEE Trans. Automatic Contr., vol. AC-30, 1985, pp. 440-446.

[15] A. Ghasemi and E. S. Sousa, "Collaborative spectrum sensing for opportunistic access in fading environments," in Proc. IEEE Dynamic Spectrum Access Nets., Baltimore, MD, 2005, pp. 131-136.

[16] D. Cabric, A. Tkachenko, and R. W. Brodersen, "Experimental study of spectrum sensing based on energy detection and network cooperation," in Proc. ACM Intl. Workshop Technol. and Policy for Accessing Spectr., Boston, MA, 2006.

[17] M. Gandetto and C. Regazzoni, "Spectrum sensing: a distributed approach for cognitive radio terminals," IEEE J. Sel. Areasa Commun., vol. 25, pp. 546-557, April 2007.

[18] J. L. Xu, W. Su, and M. Zhou, "Distributed Automatic Modulation Classification with Multiple Sensors," IEEE Sensors Journal, vol. 10, issue. 11, pp. 1779-1785, September 2010.

[19] P. A. Forero, A. Cano, and G. B. Giannakis, "Distributed Feature-based Modulation Classification using Wireless Sensor Networks", IEEE Military Communications Conference, 2008, pp. 1-7, 19 January. 2009.

[20] M. Shi, Y. Bar-Ness , and W. Su "Adaptive estimation of the number of transmit antennas,” Proc. IEEE MILCOM pp. 1-5, Oct. 2007.

[21] D. P. Bertsekas and J. N. Tsitsiklis, "Parallel and Distributed Computation: Numerical Methods”, Athena Scientific, 1997. 